\title{
Simulation study of overlap capacitance in source-gated transistors for current-mode pixel drivers
}

\author{
Raymond Drury, Eva Bestelink and Radu A. Sporea, Member, IEEE
}

\begin{abstract}
Contrary to conventional design principles, currentdriven pixel drivers based on source-gated transistors (SGTs) achieve their optimal drive current and speed with a deliberate

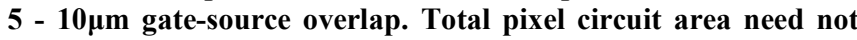
increase, as the additional device area can be compensated by reducing the pixel storage capacitor. Numerical simulations demonstrate the viability of SGTs for emissive pixel drivers and high gain, low power, robust circuits for emerging sensor arrays.
\end{abstract}

Index Terms - TFT, OLED, active matrix, display, Schottky barrier, pixel circuit, energy efficiency, layout optimization

\section{INTRODUCTION}

$\mathrm{A}$ $\mathrm{S}$ display screens continue to progress from a means of delivering information to pervasive entertainment, the competing pressures of improved image quality and low-cost fabrication will increase. Emissive, OLED-based screens show a performance advantage over competing technologies due to their high contrast [1, 2], energy efficient [3, 4], flexible [5-7] and arbitrary-shape [8-10] form factor. Significant progress has been made recently in gate driver circuit design [11-14] and complex pixel circuits, with timing strategies optimized for age compensation or areal uniformity [15-20]. Yet many practical challenges remain $[19,21]$.

Image uniformity and energy efficiency can be further improved by optimizing the pixel drive transistor itself. Source-gated transistors (SGTs) [22-25] purposely include a potential barrier at the source, as the main means of inducing current saturation. Optimized SGTs have significant advantages compared to traditional thin-film transistors (TFTs): a much lower saturation voltage [22-24], [26-29]; practical absence of short channel effects [23, 24, 30, 31]; and extremely low dependence of drain current on drain voltage [24, 26, 28, 32-34]. These properties are particularly desirable for current-driven pixels, as they enable: increased energy efficiency; more compact pixel designs for higher aperture ratios; and improved image uniformity, respectively. These devices differ marginally in their fabrication from TFTs and can be realized in practically all the usual material systems,

Submitted April 22, 2019. The work was carried out within EPSRC project $\mathrm{EP} / \mathrm{R} 028559 / 1$

The authors are with the Advanced Technology Institute, Department of Electrical and Electronic Engineering, University of Surrey, Guildford, Surrey, GU2 7XH, United Kingdom (email: r.a.sporea@surrey.ac.uk). thus being easily integrated in existing large-area processes.

The source-gate overlap required for SGT operation may be inherent in the fabrication. Even as this overlap and the unique current control mechanism lowers the maximum device speed, switching is still sufficiently fast for an OLED display pixel. An optimal range for source-gate overlap is found, which: is easily achievable in fabrication; does not reduce the dynamic performance appreciably; and incurs no significant areal penalty in the pixel circuit layout. The recommendations we derive are different to those expected for TFT circuits.

\section{DEVICE MODELING AND CHARACTERISTICS}

Device simulations were performed with the Silvaco Atlas (v. 5.19.17.C) suite. Staggered-electrode, top-gate devices have: self-aligned gate and drain electrodes; full overlap between the gate and the source over the length of the source (denoted $S$ ); a $5 \mu \mathrm{m}$ source-drain gap; a $40 \mathrm{~nm}$ semiconductor layer (a-Si or poly-Si, both using the default parameters for materials and defects from the simulator); and a $50 \mathrm{~nm} \mathrm{SiO}_{2}$ gate insulator. Aluminium was used as the ohmic drain contact. The source barrier was set by specifying the electrode work function and the barrier lowering parameter was $\alpha=$ $4 \mathrm{~nm}$.

D.c. simulations were performed to extract transfer and output characteristics. Capacitances and a.c. conductances between all pairs of terminals were computed at $f=1 \mathrm{kHz}$.

Figure 1a shows the schematic of a simple two-transistor, one capacitor (2T-1C) circuit for an OLED emissive (sub-) pixel [35]. Drive transistor, $T_{l}$, sinks current through the OLED during operation. (Sub-)pixel brightness can be unequivocally set by applying a given potential to the gate of $T_{1}$ through pass transistor $T_{2}$. When the DATA line is driven at the desired potential, opening $T_{2}$ with the SELECT signal charges capacitor $C_{S T O R E}$. Once $T_{2}$ is turned off, $C_{S T O R E}$ keeps the gate potential of $T 1$ at the desired value for the duration of the frame. Conflicting requirements intervene in the optimization of the design [36]: the emissive (aperture) area $A$ should be maximised; $C_{\text {STORE }}$ should be small enough to charge rapidly and to maximize $A$, but large enough to retain enough charge despite the leakage current of $T_{2} ; T_{2}$ should have a high on/off ratio; $T_{1}$ should occupy minimal area, but be able to source the required output current, which should not vary with drain-source voltage $\left(V_{D S}\right)$.

Here, we propose using a source-gated transistor (SGT) 

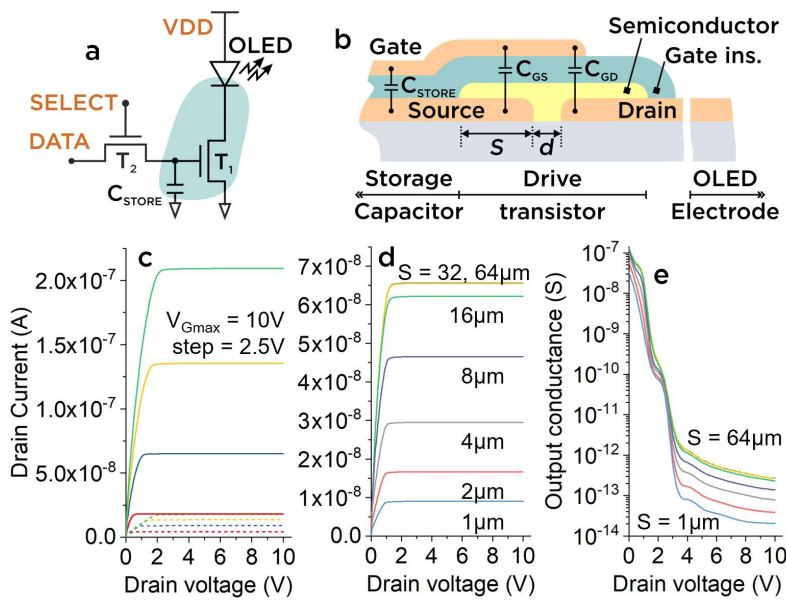

Fig. 1. a) Simple 2T-1C AMOLED pixel: $T_{l}$ - drive transistor (SGT), $T_{2}$ switch, $\mathrm{C}_{\text {STORE }}$ - storage capacitor; b) Cross-section of the shaded area in (a); c) Output curves for an $n$-type SGT with $S=32 \mu \mathrm{m}$ (solid lines) and $S=1 \mu \mathrm{m}$ (dashed lines); d) Output curve at $V_{G}=5 \mathrm{~V}$ for devices with varying $S$; e) Output conductance, $g_{d}$, plot for the curves in (d), illustrating the very low values obtained in saturation for all values of $S$.

architecture for device $T_{1}$. Despite the comparatively low transconductance, SGTs have been proven to have very low drain voltage dependence of their drain current, even at small channel lengths. This results in the possibility of having a short and wide transistor in the same layout area for increased on-current, with minimal dependence on drain voltage. Additionally, low series voltage drop $\left(V_{D S}\right)$ is essential for the drive transistor of an OLED pixel, as it enables lower supply voltages, $V_{D D}$, to be used for improved power efficiency [37], as $V_{D D}=V_{O L E D}+V_{D S(T I)}$ and $P=I_{O L E D} \times V_{D D}$.

A schematic cross-section of the devices highlighted in Figure 1a is shown in Figure 1b. Importantly, the storage capacitor exists between the electrical nodes represented by the gate and source of $T_{1}$. As for this study $T_{1}$ is an SGT, it is necessary that a certain overlap exists between the staggered gate and source [24]. This overlap increases the gate-source capacitance, $C_{G S}$, which is connected electrically in parallel with the storage capacitor. Also shown in Figure $1 \mathrm{~b}$ is the gate-to-drain (parasitic) capacitance $C_{G D}$. In practice, transistors are likely to have an overlap between the gate and the drain, contributing to $C_{G D}$. In this study, however, we have chosen a self-aligned structure, to better focus on the effects at the source. Whatever the device structure, the transistor switching speed is inversely proportional to gate capacitance.

The output curves of a-Si SGTs are shown in Figure 1c, for two source-gate overlap $(S)$ values. Of note are the low saturation voltage and flat curves in saturation, typical of SGT operation [25]. Figure 1d shows the output curve for a single $\mathrm{V}_{\mathrm{GS}}$ value for devices with increasing $S$. As shown previously $[31,38]$, for small $S$, the current increases proportionally with the source area. For larger values, this dependence reduces until it saturates (i.e. increasing $S$ further leads to no rise in drain current), explained by the resistive potential drop with distance in the accumulation layer present in the source region at the insulator interface [23, 39]. Finally, Figure 1e shows that, despite the relatively large variation of current with $S$, the output conductance has an extremely small value in saturation (below $1 \mathrm{pS} / \mu \mathrm{m}$ device width) for all values of $S$. Together with the low saturation voltage, this is an essential benefit of SGTs, which can thus be used as current sources or series drivers with reduced distortion and superior energy efficiency.

The most efficient geometry has $S$ where the dependence of the current with $S$ becomes sublinear (saturates). In the present structures this is around $S=8 \mu \mathrm{m}$ (Figure 1d). This value depends on material properties, layer thicknesses, etc., but it is significantly larger than minimum alignment errors and tolerances in large-area fabrication. $S=5-10 \mu \mathrm{m}$ should not pose any problems to contemporary thin-film technologies.

Transfer characteristics for a-Si transistors are shown in Figure 2a. For the same geometry, the conventional ohmic contact TFT will have higher current than the SGT, for which the on-current depends on $S$ until saturation, as explained above. The d.c. characteristics of the TFT are, of course, independent of source length. The subthreshold region of both TFTs and SGTs is controlled by the semiconductor and is of no interest to this study. Transfer curves for polysilicon SGTs are presented in Figure 2b. Subthreshold behavior differs from the a-Si SGTs in Figure 1a due to altered material properties.

Current levels are comparable between poly-Si and a-Si devices for a given source work function, however, poly-Si SGT transconductance is somewhat higher due to the greater carrier mobility, a second-order effect in SGTs. Lowering the barrier to $4.52 \mathrm{eV}$ raises the current and reduces dependence on $S$. The higher current is a direct result of the lower barrier, while the lower value of $S$ for which current saturates with $S$ is attributed to higher resistive potential drop in the semiconductor, and thus a reduction of the contribution to drain current from the bulk of the source [23, 39].

\section{FREQUENCY BEHAVIOR VS. DEVICE GEOMETRY}

The most efficient geometry for d.c. current output has $S \approx$ $8 \mu \mathrm{m}$. Concerning dynamic performance, the gate-source overlap increases gate capacitance, and thus reduces the frequency response. Figure $2 \mathrm{c}$ shows the proportion of total gate capacitance represented by $\mathrm{C}_{\mathrm{GS}}$ for various $S$ and source work function. A-Si and poly-Si devices with self-aligned gate/drain and $d=5 \mu \mathrm{m}$ are shown. Usual design directions would suggest that $S$ should be minimized for fast operation (e.g. in Figure 3, for $S>8 \mu \mathrm{m}, C_{G S}$ is $>80 \%$ of gate
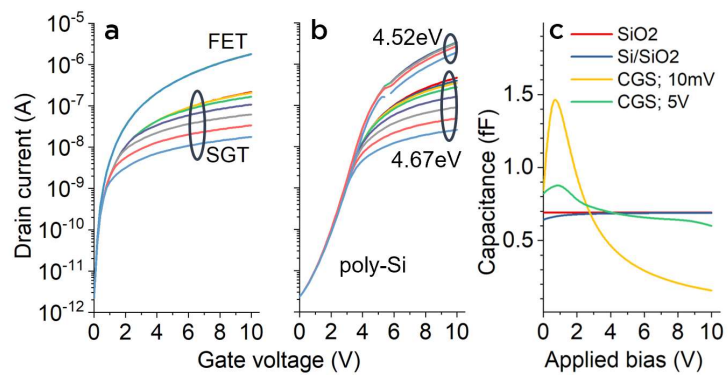

Fig. 2. Transfer curves for: a) a-Si:H SGTs and TFT FETs $(S=0,4,12 \mu \mathrm{m}$ for TFT; 1, 2, 4, 8, 16, 32, 64 $\mu \mathrm{m}$ for SGT) and b) poly-Si SGTs with the same $S$ as in (a), varying source work function; c) Capacitance vs. applied bias, in a $5 \mu \mathrm{m} \times 1 \mu \mathrm{m}$ area of interest for: $\mathrm{a} \mathrm{SiO}_{2}$ capacitor; a $\mathrm{Si} / \mathrm{SiO}_{2}$ structure with the same Schottky contact as in the SGT; a SGT at two values of $\mathrm{V}_{\mathrm{D}}$, plotting gate to source capacitance and gate-source voltage representing applied bias. 
capacitance). Two SGT operating characteristics recommend that source-gate overlap be larger than in conventional TFTs.

First, in the pixel circuit, the structure formed by the source, semiconductor, gate insulator and gate is connected in parallel with the $C_{\text {STORE. }}$. From Figure 2c, it can be observed that the gate-source capacitance of a SGT operated in saturation is similar to that of a MIM or a MIS capacitor of the same area, due to the accumulated charge layer at the semiconductorinsulator interface $[23,38,39]$ in this bias condition. The drive transistor's $C_{G S}$ can thus be used as part of the required storage capacitance with practically the same areal efficiency as a MIM capacitor. Using SGTs, even with their required gatesource overlap, will not compromise pixel layout.

Second, in OLED displays, fast switching of $T_{1}$ is not required, but optimization is desirable. SGT drain current $I_{D}$ increases initially proportionally with $S$, then saturates as $S$ increases. Concurrently, the gate-source capacitance $C_{G S}$ of the transistor is to a good approximation proportional to $S$, and the $C_{G S}$ is itself the dominant term in the total gate capacitance $C_{G}$. We can consider cut-off frequency $f_{T}=g_{m} /\left(2 \pi C_{G}\right)$ as a figure of merit representative of switching speed, where $g_{m}=$ $d I_{D} / d V_{G}$ is the transconductance.

There is then a range of optimal $S$ where $f_{T}$ is maximised, intuitively at values of $S$ beyond which the current dependence on $S$ becomes sublinear (while the $C_{G S}$ keeps increasing in proportion to $S$ ). Figure 4 shows the extracted values for $f_{T}$ at different bias conditions for the a-Si SGT, poly-Si SGT and aSi TFT, respectively. Both SGTs show an initial increase of $f_{T}$ with $S$, then $f_{T}$ reduces, as the additional capacitance introduced by larger $S$ has a greater contribution to $f_{T}$ than the increased $g_{m}$. Both technologies show a peak in $f_{T}$ around $S=$ $8 \mu \mathrm{m}$, with the higher absolute value reached by the poly-Si devices attributed to higher carrier mobility. The TFT exhibits a completely different behavior. For no value of $S$ greater than zero does $f_{T}$ improve, since current does not change with $S$ and gate-source overlap increases gate capacitance, lowering $f_{T}$. The TFT's $f_{T}$ is 5-30 times higher than that of the SGTs depending on bias and $S$, due to its larger $g_{m}$ in the absence of the source barrier. Even so, the SGT's $>250 \mathrm{kHz} f_{T}$ is ample for this application. Figure 4 also confirms the SGT's tolerance to geometrical variations. In high-throughput printing, alignment errors may occur, yet the SGT's $f_{T}$ is practically unchanged
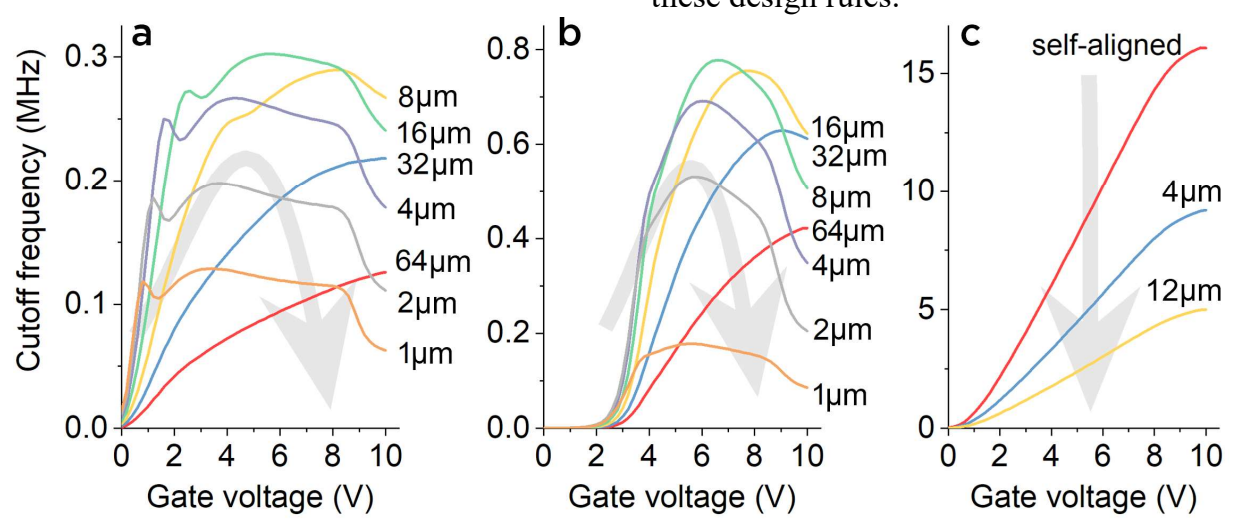
is also insensitive to $S$ for large $S$ (see Figure 1d).

\section{CONCLUSIONS} improved bias stress stability. these design rules.

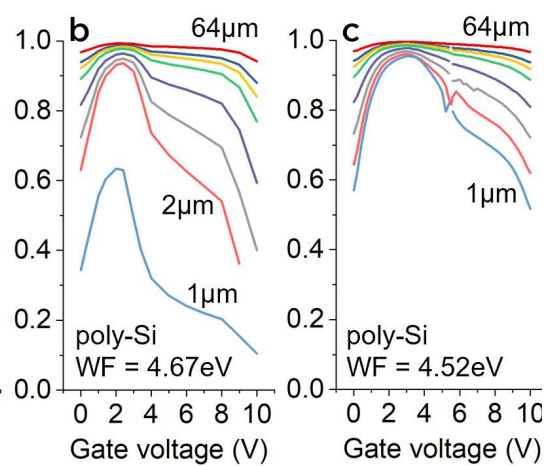

Fig. 3. Gate-to-source capacitance as a proportion of total gate capacitance and its dependence on $S$ for SGTs modelled in different materials and with different values of the source metal work function. The gate capacitance is dominated by the gate-source component even at comparatively small values of $S$. While overlap capacitance increases linearly with $S$, total drain current eventually saturates due to the two-dimensional injection at the source.

over a large range of $S(4-16 \mu \mathrm{m}$ in Figure 4a). Drain current

With flat output curves for reduced non-linearity and low saturation voltage for power efficiency, the properties shown here make SGTs robust, versatile drivers for emissive pixels.

SGTs offer significant advantages to current driver circuits: the low saturation drain voltage can potentially reduce power consumption by allowing a lower supply voltage to be used. In emissive pixel circuits, SGT drivers can improve the uniformity of light emission through: low drain-voltage dependence of drain current; tolerance to geometrical variations during processing; and (e.g. in a-Si:H and $\mathrm{ZnO}$ ),

To fully exploit SGT features, circuit design techniques require adaptation. Contrary to conventional rules, SGTs acting as drive transistors in current-mode drivers benefit from a source-gate overlap of several microns for increased current injection and optimal speed. When the transistor is used in a pixel driver circuit, its source-gate overlap also acts as a storage capacitor, and its area substitutes a portion of the usual MIM capacitor, with potentially no increase of total circuit size. Arrays of precision low-power analog circuits for emerging flexible sensors should find similar benefits from

Fig. 4. Calculated cut-off frequency $\left(f_{T}\right)$ and its dependence on gate bias and source-gate overlap for a) an a-Si:H SGT with source contact work function $\mathrm{WF}=4.67 \mathrm{eV} ; \mathrm{b}$ ) a poly-Si SGT with $\mathrm{WF}=4.67 \mathrm{eV} ; \mathrm{c}$ ) an a-Si:H TFT with ohmic contacts. The optimal source-gate overlap is zero for the TFT, but in the range of $4-16 \mu \mathrm{m}$ for the SGT as a result of the interplay between transconductance and gate capacitance. 


\section{REFERENCES}

[1] S. Okutani, M. Kobayashi, and N. Ibaraki, "Quantitative evaluation of display characteristics of AMOLED displays," J. Soc. Inf. Disp., vol. 14, no. 12, pp. 1119-1125, Dec. 2006, doi: 10.1889/1.2408395.

[2] J. J. Lih and T.H. Wang, "A true circular 1.39 inch AMOLED for wearable application," SID Symp. Dig. Tech. Pap., vol. 47, no. 1, pp. 566-569, May 2016, doi: 10.1002/sdtp.10730.

[3] Soeren Steudel, J.-L. van der Steen, M. Nag, T. H. Ke, S. Smout, T. Bel, K. van Diesen, G. de Haas, J. Maas, J. de Riet, M. Rovers, R. Verbeek, Y.-Y. Huang, S.-C. Chiang, M. Ameys, F. De Roose, et al., "Power saving through state retention in IGZO-TFT AMOLED displays for wearable applications," SID Symp. Dig. Tech. Pap., vol. 48, no. 1, pp. 38-41, May 2017, doi: 10.1002/sdtp.11575

[4] T. -H. Wang, K. Liu, Y.-Y. Dai, C. Yeh, W. R. Chen, C. Tsao, Y.-H Li, L.-W. Shih, and J. J. Lih, "Ultra low power AMOLED for wearable application," SID Symp. Dig. Tech. Pap., vol. 48, no. 1, pp. 1919-1921, May 2017, doi: 10.1002/sdtp.12005.

[5] N. Münzenrieder, P. Voser, L. Petti, C. Zysset, L. Büthe, C. Vogt, G. A. Salvatore, and G. Tröster, "Flexible self-aligned double-gate IGZO TFT," IEEE Electron Device Lett., vol. 35, no. 1, pp. 69-71, 2014, doi: 10.1109/LED.2013.2286319.

[6] J. Lee, T. T. Nguyen, J. Bae, G. Jo, Y. Lee, S. Yang, H. Chu, and J. Kwag, "5.8-inch QHD flexible AMOLED display with enhanced bendability of LTPS TFTs," J. SID, vol. 26, no. 4, pp. 200-207, 2018, doi: $10.1002 /$ jsid. 655 .

[7] X. Gao, L. Lin, Y. Liu, and X. Huang, "LTPS TFT process on polyimide substrate for flexible AMOLED," J. Disp. Technol., vol. 11, no. 8, pp. 666-669, 2015, doi: 10.1109/JDT.2015.2419656

[8] J.-H. Hong, J. M. Shin, G. M. Kim, H. Joo, G. S. Park, I. B. Hwang, M. W. Kim, W.-S. Park, H. Y. Chu, and S. Kim, "9.1-inch stretchable AMOLED display based on LTPS technology," J. Soc. Inf. Disp., vol. 25, no. 3, pp. 194-199, Mar. 2017, doi: 10.1002/jsid.547.

[9] W. Fan, J. Cheng, X. Zhu, Y. Du, and X. Huang, "The application of AMOLED in smart wearable technology," SID Symp. Dig. Tech. Pap., vol. 49, no. S1, pp. 212-213, Apr. 2018, doi: 10.1002/sdtp.12684.

[10] F. Yuan, G. Zhang, Q. Ma, P. Sun, Y. Yu, R. Zhang, D.K. Yoon, and Z. Li, "Automotive display innovation: 12.3-inch automotive freeform curved cluster," J. Soc. Inf. Disp., vol. 25, no. 1, pp. 61-64, Jan. 2017, doi: 10.1002/jsid.525.

[11] D. Geng, Y. F. Chen, M. Mativenga, and J. Jang, "30 4 m-pitch oxide TFT-based gate driver design for small-size, high-resolution, and narrow-bezel displays," IEEE Electron Device Lett., vol. 36, no. 8, pp. 805-807, 2015, doi: 10.1109/LED.2015.2445319.

[12] S. Moon, I. Haruhisa, K. Kim, C.-W. Park, H.-J. Chung, S.-H. Kim, B.-K. Kim, and O. Kim, "Highly robust integrated gate-driver for incell touch TFT-LCD driven in time division driving method," J. Disp. Technol., vol. 12, no. 5, pp. 435-441, 2016, doi: 10.1109/JDT.2015.2495121.

[13] W.-C. Chiu, C.-C. Hsu, P.-C. Lai, M.-X. Wang, and C.-L. Lin, "Novel a-Si:H gate driver circuit with high charging and discharging speeds for use in high-resolution liquid-crystal displays," in Proc AM-FPD 2018 , pp. 1-4, 2018, doi: 10.23919/AM-FPD.2018.8437402 .

[14] C.-L. Lin, P.-C. Lai, P.-C. Lai, T.-C. Chu, and C.-L. Lee, "Bidirectional gate driver circuit using recharging and time-division driving scheme for in-cell touch LCDs," IEEE Trans. Ind. Electron., vol. 65, no. 4, pp. 3585-3591, 2018, doi: 10.1109/TIE.2017.2756583.

[15] C.-L. Lin, C.-C. Hung, P.-S. Chen, P.-C. Lai, and M.-H. Cheng, "New voltage-programmed AMOLED pixel circuit to compensate for nonuniform electrical characteristics of LTPS TFTs and voltage drop in power line," IEEE Trans. Electron Devices, vol. 61, no. 7, pp. 2454 2458, Jul. 2014, doi: 10.1109/TED.2014.2325612.

[16] C.-H. Ho, C. Lu, and K. Roy, "An enhanced voltage programming pixel circuit for compensating GB-induced variations in poly-Si TFTs for AMOLED displays," J. Disp. Technol., vol. 10, no. 5, pp. 345-351, May 2014, doi: 10.1109/JDT.2014.2301020.

[17] K.-Y. Lee, Y.-P. Hsu, P. C.-P. Chao, and W.-D. Chen, "A new compensation method for emission degradation in an AMOLED display via an external algorithm, new pixel circuit, and models of prior measurements," J. Disp. Technol., vol. 10, no. 3, pp. 189-197, Mar. 2014, doi: 10.1109/JDT.2013.2292555.

[18] C. Liao, W. Deng, D. Song, S. Huang, and L. Deng, "Mirrored OLED pixel circuit for threshold voltage and mobility compensation with IGZO TFTs," Microelectronics J., vol. 46, no. 10, pp. 923-927, Oct. 2015, doi: 10.1016/j.mejo.2015.08.003.
[19] C.-L. Lin, P.-S. Chen, M.-Y. Deng, C.-E. Wu, W.-C. Chiu, and Y.-S Lin, "UHD AMOLED driving scheme of compensation pixel and gate driver circuits achieving high-speed operation," IEEE J. Electron Devices Soc., vol. 6, pp. 26-33, 2018, doi: 10.1109/JEDS.2017.2763601.

[20] C.-L. Fan, Y.-C. Chen, C.-C. Yang, Y.-K. Tsai, and B.-R. Huang, "Novel LTPS-TFT pixel circuit with OLED luminance compensation for 3D AMOLED displays," J. Disp. Technol., vol. 12, no. 5, pp. 425428, 2016, doi: 10.1109/JDT.2016.2525736.

[21] G. S. Ryu, J. S. Kim, S. H. Jeong, and C. K. Song, "A printed OTFTbackplane for AMOLED display," Org. Electron., vol. 14, no. 4, pp. 1218-1224, 2013, doi: 10.1016/j.orgel.2013.02.006.

[22] J. M. Shannon and E. G. Gerstner, "Source-gated thin-film transistors," Electron Device Lett. IEEE, vol. 24, no. 6, pp. 405-407, 2003, doi: 10.1109/LED.2003.813379.

[23] A. Valletta, L. Mariucci, M. Rapisarda, and G. Fortunato, "Principle of operation and modeling of source-gated transistors," J. Appl. Phys., vol. 114, no. 6, p. 64501, 2013, doi: 10.1063/1.4817502.

[24] R. A. Sporea, M. J. Trainor, N. D. Young, J. M. Shannon, and S. R. P. Silva, "Intrinsic gain in self-aligned polysilicon source-gated transistors," Electron Devices, IEEE Trans., vol. 57, no. 10, pp. 2434 2439, 2010, doi: 10.1109/TED.2010.2056151.

[25] S. D. Brotherton, Introduction to Thin Film Transistors: Physics and Technology of TFTs. Springer, 2013, ISBN 978-3-319-00002-2.

[26] J. M. Shannon and E. G. Gerstner, "Source-gated transistors in hydrogenated amorphous silicon," Solid. State. Electron., vol. 48, no. 7 , pp. 1155-1161, 2004, doi: 10.1016/j.sse.2004.02.004.

[27] A. M. Ma, M. Gupta, F. R. Chowdhury, M. Shen, K. Bothe, K. Shankar, Y.Tsui and D. W. Barlage, "Zinc oxide thin film transistors with Schottky source barriers," Solid. State. Electron., vol. 76, pp. 104 108, 2012, doi: 10.1016/j.sse.2012.05.005.

[28] L. Wang, Y. Sun, X. Zhang, L. Zhang, S. Zhang, and M. Chan, "Tunneling contact IGZO TFTs with reduced saturation voltages," Appl. Phys. Lett., vol. 110, no. 15, p. 152105, Apr. 2017, doi: 10.1063/1.4980131.

[29] C. Opoku, R. A. Sporea, V. Stolojan, S. R. P. Silva, and M. Shkunov, "Source-gated transistors based on solution processed silicon nanowires for low power applications," Adv. Electron. Mater., vol. 3, no. 1, p. 1600256, 2016, doi: 10.1002/aelm.201600256.

[30] S. Lee and A. Nathan, "Subthreshold Schottky-barrier thin-film transistors with ultralow power and high intrinsic gain," Science, vol. 354 , no. 6310 , p. 302 , 2016, doi: 10.1126/science.aah5035.

[31] R. A. Sporea, G. Xiaojun, J. M. Shannon, and S. R. P. Silva, "Effects of process variations on the current in Schottky barrier source-gated transistors," in Proc CAS 2009, vol. 2, pp. 413-416, 2009, doi: 10.1109/SMICND.2009.5336693.

[32] R. A. Sporea, M. J. Trainor, N. D. Young, J. M. Shannon, and S. R. P. Silva, "Field plate optimization in low-power high-gain source-gated transistors," Electron Devices, IEEE Trans., vol. 59, no. 8, pp. 2180 2186, 2012, doi: 10.1109/TED.2012.2198823

[33] R. A. Sporea, M. J. Trainor, N. D. Young, J. M. Shannon, and S. R. P. Silva, "Source-gated transistors for order-of-magnitude performance improvements in thin-film digital circuits,"Sci. Rep., vol. 4, 2014, doi: 10.1038/srep04295.

[34] A. S. Dahiya, C. Opoku, R. A. Sporea, B. Sarvankumar, G. PoulinVittrant, F. Cayrel, N. Camara, and D. Alquier, "Single-crystalline ZnO sheet source-gated transistors," Sci. Rep., vol. 6, p. 19232, 2016, doi: 10.1038/srep19232.

[35] A. Nathan, G. R. Chaji, and S. J. Ashtiani, "Driving schemes for a-Si and LTPS AMOLED displays," J. Disp. Technol., vol. 1, no. 2, pp 267-277, 2005, doi: 10.1109/JDT.2005.858913.

[36] C.-W. Lin, M. C.-T. Chao, and Y.-S. Huang, "A novel pixel design for AM-OLED displays using nanocrystalline silicon TFTs," IEEE T. VLSI Syst., vol. 19, no. 6, pp. 939-952, 2011, doi: 10.1109/TVLSI.2010.2042735.

[37] X. Xu, R. A. Sporea, and X. Guo, "Source-gated transistors for powerand area-efficient AMOLED pixel circuits," J. Disp. Tech., vol. 10, no. 11, pp. 928-933, 2014, doi: 10.1109/JDT.2013.2293181

[38] R. A. Sporea and S. R. P. Silva, "Design considerations for the source region of Schottky-barrier source-gated transistors," in Proc CAS 2017 , pp. 155-158, 2017, doi: 10.1109/SMICND.2017.8101185.

[39] J. M. Shannon, R. A. Sporea, S. Georgakopoulos, M. Shkunov, and S. R. P. Silva, "Low-field behavior of source-gated transistors", IEEE Trans. Electron. Dev., vol. 60, no. 8, pp. 2444-2449, 2013, doi: $10.1109 /$ TED.2013.2264547. 